\title{
A Robust ECG Denoising Technique Using Variable Frequency Complex
}

\section{Demodulation}

\author{
Md-Billal Hossain ${ }^{1}$, Syed Khairul Bashar ${ }^{1}$, Jesus Lazaro ${ }^{2}$, Natasa Reljin ${ }^{1}$, Yeonsik Noh ${ }^{3}$, and Ki H. Chon ${ }^{1, *}$ \\ ${ }^{1}$ Department of Biomedical Engineering, University of Connecticut, 260 Glenbrook Road, Unit 3247 Storrs, CT 06269-3247, USA \\ ${ }^{2}$ Aragon Institute for Engineering Research, University of Zaragoza (Spain) \\ ${ }^{3}$ Department of Electrical and Computer Engineering at University of Massachusetts Amherst. \\ *Corresponding Author. Email: ki.chon@uconn.edu
}

\begin{abstract}
Background and Objective: Electrocardiogram (ECG) is widely used for the detection and diagnosis of cardiac arrhythmias such as atrial fibrillation. Most of the computer-based automatic cardiac abnormality detection algorithms require accurate identification of ECG components such as QRS complexes in order to provide a reliable result. However, ECGs are often contaminated by noise and artifacts, especially if they are obtained using wearable sensors, therefore, identification of accurate QRS complexes often becomes challenging. Most of the existing denoising methods were validated using simulated noise added to a clean ECG signal and they did not consider authentically noisy ECG signals. Moreover, many of them are model-dependent and sampling-frequency dependent and require a large amount of computational time.
\end{abstract}

Methods: This paper presents a novel ECG denoising technique using the variable frequency complex demodulation (VFCDM) algorithm, which considers noises from a variety of sources. We used the sub-band decomposition of the noise-contaminated ECG signals using VFCDM to remove the noise components so that better-quality ECGs could be reconstructed. An adaptive automated masking is proposed in order to preserve the QRS complexes while removing the unnecessary noise components. Finally, the ECG was reconstructed using a dynamic reconstruction rule based on automatic identification of the severity of the noise contamination. The ECG signal quality was further improved by removing baseline drift and smoothing via adaptive mean filtering. 
Results: Evaluation results on the standard MIT-BIH Arrhythmia database suggest that the proposed denoising technique provides superior denoising performance compared to studies in the literature. Moreover, the proposed method was validated using real-life noise sources collected from the noise stress test database (NSTDB) and data from an armband ECG device which contains significant muscle artifacts. Results from both the wearable armband ECG data and NSTDB data suggest that the proposed denoising method provides significantly better performance in terms of accurate QRS complex detection and signal to noise ratio (SNR) improvement when compared to some of the recent existing denoising algorithms.

Conclusions: The detailed qualitative and quantitative analysis demonstrated that the proposed denoising method has been robust in filtering varieties of noises present in the ECG. The QRS detection performance of the denoised armband ECG signals indicates that the proposed denoising method has the potential to increase the amount of usable armband ECG data, thus, the armband device with the proposed denoising method could be used for long term monitoring of atrial fibrillation.

Keywords: VFCDM, ECG, armband, QRS complex, EMG, AWGN, PLI.

\section{Introduction}

The electrocardiogram (ECG) has been widely used for detection and classification of different life-threatening cardiac arrhythmias. For accurate detection of cardiac abnormalities, ECG signals must retain their morphological components in order to provide reliable and essential information about cardiac activity. However, ECGs are most often contaminated by a wide variety of noise sources including motion artifacts, bad electrode contact to the skin, and muscle and power line interference that can distort the ECG morphologies and lead to misdetection of cardiac arrhythmias. Noise is more acute in ECGs from wearable devices simply because of movement artifacts. Therefore, it is very important and necessary to remove noise and artifacts from ECG signals in order to increase the usability of ECGs. However, denoising ECG signals is very challenging, especially if the noise frequency overlaps with the signal's frequency. There have been several denoising methods proposed in the literature in the last few decades. The most well-developed methods are based on discrete wavelet transform (DWT) decomposition [1]-[3], adaptive 
filtering [4], [5], empirical mode decomposition (EMD) and ensemble empirical mode decomposition (EEMD) [6][9], FIR filtering [10], [11], Kalman filtering [12], principal component analysis (PCA) [13], independent component analysis (ICA) [14], nonlocal means (NLM) [15], and neural networks [16], [17]. While many methods showed promising denoising performance, they have their own advantages and disadvantages.

The wavelet-based approaches [1]-[3] use soft and hard thresholding, which are popular for denoising nonstationary signals. However, wavelet-based methods cannot preserve the edges and are sensitive to intra-subject and inter-subject variations. Adaptive filtering approaches [4], [5] are often used for removing electromyogram (EMG) and motion artifacts. The main drawback of an adaptive filtering approach is that it requires a reference signal which is not often available.

The EMD-based approaches [6], [7] performed better when compared to wavelet-based thresholding methods, but they cannot completely remove noise. An adaptive switching mean filtering was proposed with EMD and DWT in [8] and a better result was shown when compared with previous EMD-based approaches. However, this approach needs an external QRS detector to preserve the QRS complexes. In addition, the EMD has a mode mixing problem [18]; as a result, the intrinsic mode functions (IMFs) extracted can be incorrect, decreasing the denoising performance.

The Kalman filtering approaches for denoising [12] are effective in preserving the edges of the signal. However, they require manual initialization of the parameters that are associated with the amplitude, width, and phase of the components of a complete ECG cycle. The PCA [13] and neural network [16] approaches require multiple leads to obtain better denoising performance using correlation. However, their performance is suboptimal for a single lead ECG. For ICA-based denoising [14], visual inspection of the independent components is essential, but this is not feasible for long-term applications. The non-local means (NLM) [15] method is a popular denoising technique that provides improvements in SNR. The main disadvantage of this approach is that its performance depends on the choice of a parameter's bandwidth, which depends on the noise standard deviation that might not be available in real time.

Some new ECG denoising techniques have been proposed in recent years such as the variational mode decomposition (VMD) method [19] and adaptive Fourier decomposition (AFD) [20]. An eigenvalue decompositionbased denoising is proposed in [21], where eigenvalue decomposition of the Hankel matrix approach is used for the baseline drift and powerline interference removal. A multi-lead model-based ECG signal denoising with an adaptive guided filter is proposed in [22]. Finally, convolutional encoder-decoder approaches were proposed in [17], [23]. 
However, the performance of these methods has not been compared with existing methods. Moreover, the deep learning-based approaches function as a black box, which requires more data for training and can be computationally expensive. Therefore, deep learning based denoising may not be suitable in real time scenarios, especially for wearable device applications.

Most of the previously developed denoising methods were not validated using the realistic scenario of motion artifacts and muscle noise data; rather, they were tested on synthetically generated additive white Gaussian noise (AWGN) or random noise that were added to clean ECG signals.

In this paper, we propose a novel ECG denoising technique using the variable frequency complex demodulation (VFCDM) decomposition algorithm [24]. While a preliminary study of this method was published in the IEEE EMBC conference proceedings [25], this paper contains detailed results with a slight modification of parameters. We used the sub-band decomposition of a noisy ECG signal via the VFCDM to reconstruct a cleaner ECG signal by removing subcomponents that are associated with noise dynamics. Finally, we removed the baseline drift and abrupt noise components to achieve the final desired denoised signal. The performance of the proposed denoising technique was validated on the MIT-BIH arrhythmia database and the noisy ECG data obtained using a wearable armband device which was developed in the Chon lab [26]. We considered different types of noise sources both real and simulated to validate the proposed denoising technique. The simulated noises were used to compare the denoising performance with the existing denoising techniques since most of the denoising methods were validated using simulated noise. We used additive Gaussian white noise (AWGN), colored noise (blue, pink and violet), and powerline interference (PLI) to contaminate the ECGs and compared denoising performances of the proposed method with some of the existing denoising techniques. Moreover, the proposed method was tested using different real-life noisy conditions such as baseline wander, muscle noise, and electrode noises collected from the noise stress test database (NSTDB) [27]. Finally, the proposed method was successfully applied on the wearable armband ECG data corrupted by substantial amount of muscle artifact and the algorithm's performance was compared with some of the existing denoising techniques.

The rest of the paper is organized as follows: in the Materials and Methods section, we describe our dataset and present our proposed denoising algorithm. The performance of the proposed denoising technique is discussed in the Results section and perspective is provided in the Discussion section. Finally, a summary of the outcomes of our work is provided in the Conclusions section. 


\section{Materials and Methods}

\subsection{Description of Datasets}

We considered ECG recordings from MIT-BIH arrhythmia database (MITDB), and our own wearable armband ECG database [26] to validate our proposed denoising technique. In addition, we collected the real noise sources from MIT-BIH noise stress test database (NSTDB) to contaminate the ECG records. The datasets are described below.

\section{A) MIT BIH Arrhythmia Database}

The MIT-BIH arrhythmia database is a well-known publicly available database. It consists of 48 half-hour long datasets of two-channel ambulatory ECG recordings which were obtained from 47 different subjects [27], [28]; 25 out of the 47 patients were male aged 32 to 89 , and 22 were female aged 22 to 89 . This database contains ECGs with a variety of waveforms and different abnormalities such as complex ventricular, junctional, and supraventricular arrhythmias and conduction abnormalities. The ECG recordings were digitized with a sampling frequency of $360 \mathrm{~Hz}$ and resolution of 11 bits over an $11 \mathrm{mv}$ range. Each recording consists of two leads, one of which is modified limb lead II and the other was any of the following: V1, V2, V4, or V5.

\section{B) Wearable Armband ECG Data}

The armband ECG data were collected using a wearable armband device which has been developed in our lab at the University of Connecticut [26], [29]. This device has three pairs of hydrophobic electrodes, also developed in our lab [30], which are sequentially arranged for recording three different ECG channels. Because of the hydrophobic electrodes and the ease of wearability, this device is more comfortable than a typical Holter monitor that uses obtrusive leads and wet electrodes which are known to cause skin irritation. ECGs were obtained continuously for 24 hours with the armband worn on the upper left arm. The database contains 24 hours of continuous ECG recordings from each of the 16 healthy subjects aged $27.56 \pm 8.82$ years (mean \pm standard deviation). The subjects were instructed to carry out their regular activities but without any intense exercise. In addition to the armband ECG, a simultaneous reference 
ECG was recorded with a very widely available Holter monitor (Rozinn RZ 153+, Glendale, NY, USA). All ECGs were obtained at a sampling frequency of $1000 \mathrm{~Hz}$ which were then down-sampled to $256 \mathrm{~Hz}$.

\section{(C) The MIT-BIH Noise Stress Test Database}

The MIT-BIH Noise Stress Test Database consists of 12 half hour ECG recordings and 3 half-hour recordings of noise in typical ambulatory ECG recordings [27], [28]. The noises include baseline wander, muscle artifact and electrode motion artifact which were made using physically active volunteers and standard ECG recorders, leads, and electrodes; the electrodes were placed on the limbs in positions in which subjects' ECGs were not visible. Calibrated amounts of different levels of noise were added to the two clean ECG recordings $(118,119)$ from the MIT-BIH Arrhythmia Database. Different levels of noise were added to each of the recordings to make 6 different signals with different signal-to-noise ratios (SNRs) $(24,18,12,6,0$, and $6 \mathrm{~dB})$. All the ECG signals were sampled with a sampling frequency of 360 samples per second.

\subsection{VFCDM Decomposition of Noisy ECG Signals}

VFCDM is a high-resolution time-frequency analysis technique [24] that has been used for a variety of physiological signal processing [31]-[33]. While providing a high-resolution time-frequency spectrum (TFS), VFCDM also retains accurate amplitude distribution of the signal. Using VFCDM, we decomposed the noisy ECG into $N_{c}(=12)$ number of modes or subbands.

$$
Y_{n}(t)=\sum_{i=1}^{N_{c}} C_{i}(t)
$$

In this equation, $Y_{n}(t)$ is the noisy ECG signal, $C_{i}$ represents the $i_{t h}$ frequency component, and $N_{c}$ is the total number of subbands components (here, $N_{c}=12$ ). In this study, we decomposed the noisy ECG signal into 12 nonoverlapping frequency bands using VFCDM. Given that the sampling frequency of the ECG was $360 \mathrm{~Hz}$, the subband frequencies were equally spaced between 0 and $180 \mathrm{~Hz}$. 


\subsection{Signal Reconstruction}

Fig. 1 shows an ECG segment of 2000 samples on which additive Gaussian white noise (AWGN) with a 20 dB signal-to-noise ratio level was superimposed, and its 12 VFCDM sub-band components. As can be seen from the figure, the frequency components after the $4 t h$ sub-band are noisy, with low amplitudes. It is also observable from Fig. 1 that most of the ECG components, especially the $\mathrm{P}$ and $\mathrm{T}$ waves, are retained in the first component $C_{1}$. However, the QRS complex is attenuated and relatively wider than in the original ECG shown in Fig. Therefore, higher-frequency components (2-4) mostly contribute to the QRS complexes. We used only the first 4 components for our signal reconstruction.

To summarize again, the first component $C_{1}$ preserves most of the ECG information and components 2 to 4 contribute to the morphology of the QRS complex. To reconstruct the de-noised signal, we compute an automated mask that preserves the values around only a small window of the QRS complex. We multiply components 2 to 4 by that mask to add them to the first component to obtain the final reconstructed signal. We start with reconstructing an intermediate signal, $\breve{Y}(t)$, using the first two components. We perform a soft thresholding-based wavelet [2] denoising on the second component and added it to the first component to obtain an intermediate ECG signal, $\breve{Y}(t)$.

$$
\breve{Y}(t)=\mathrm{C}_{1}(t)+\widetilde{C_{2}(t)}
$$

$\widetilde{C_{2}(t)}$ represents the wavelet-denoised $C_{2}(t)$. Next, we compute an automatic threshold using an approach similar to the one described in [34], [35]. A histogram is computed from the local maxima of $\breve{Y}(t)$, from which the centroid is calculated using the following equation:

$$
r=\frac{\sum_{i=1}^{N} x_{i} y_{i}}{\sum_{j=1}^{N} y_{j}}
$$

where $x_{i}$ is the signal magnitude, $y_{i}$ is the distribution value of the signal, and $r$ is the centroid of the histogram. Based on the centroid, we define a threshold, $t h=\alpha r$, where $\alpha$ is a multiplying factor (in this paper we used $\alpha=1$ ). Once the threshold is calculated, we determine all local maxima that satisfy the threshold criterion. As mentioned in 
[35], these local maxima correspond to the QRS complex peaks. The QRS complex peaks are then used to create a mask, $m(t)$, which is defined as follows:

$$
m(t)=\left\{\begin{array}{cc}
\operatorname{Tukey}(N, \gamma), & R(i)-\epsilon \leq t \leq R(i)+\epsilon \\
0, & \text { elsewhere }
\end{array}\right.
$$

where $\operatorname{Tukey}(N, \gamma)$ stands for a tapered Tukey window of length $N(=50)$ with the tapering parameter $\gamma, R(i)$ represents the $i_{t h}$ QRS complex position, $i$ varies from 1 to the total number of QRS complexes $\left(N_{R}\right)$, and the $\epsilon$ determines the spread of the Tukey window from the R peak. Depending on the noise level, the 2nd component $C_{2}(t)$ of VFCDM can be either noisy or clean. In the case of a less-noisy ECG signal, $C_{2}(t)$ will also be mildly affected by

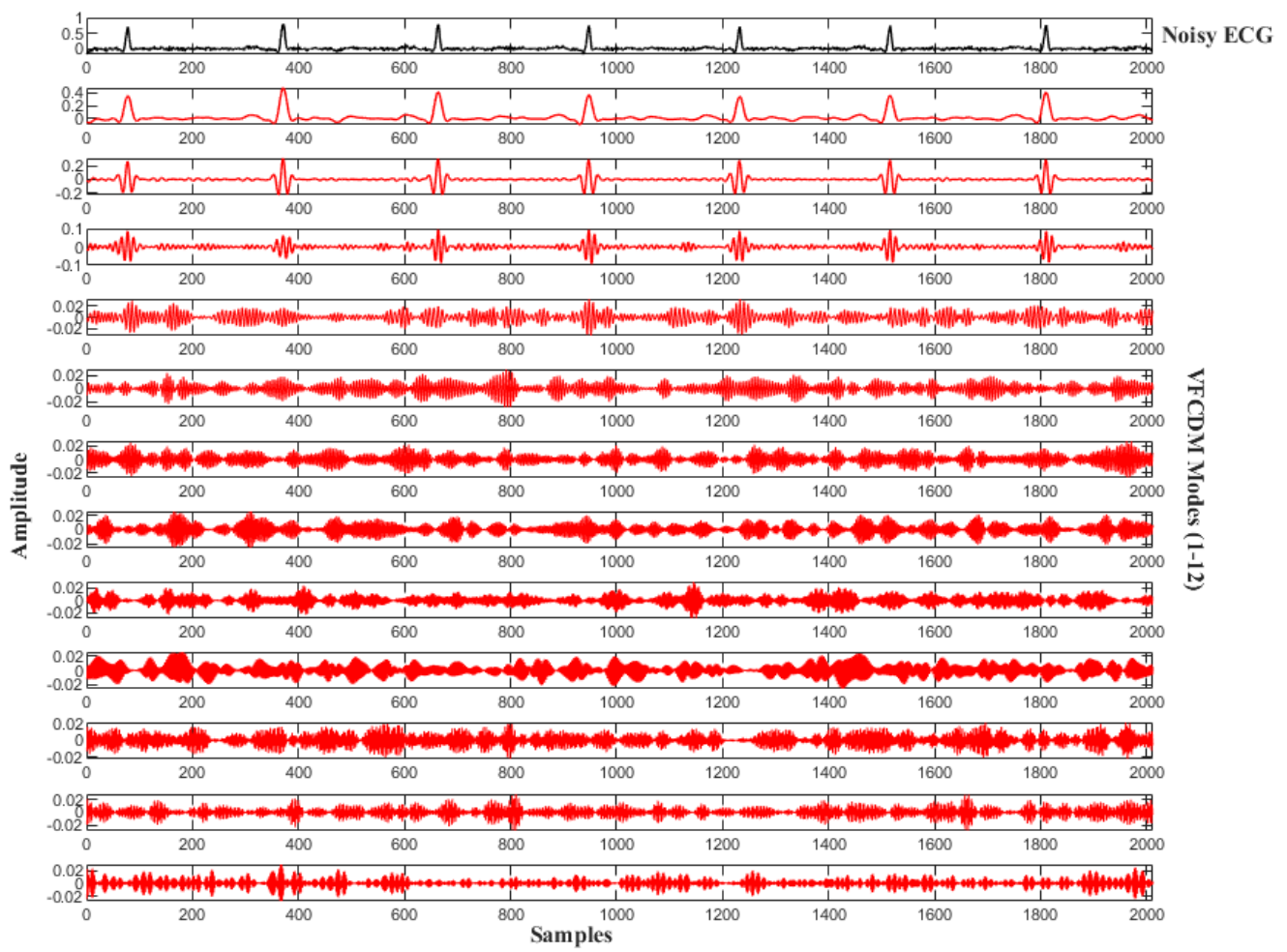

Fig. 1. VFCDM decomposition of a noisy ECG segment: a noisy ECG signal with a $20 \mathrm{~dB}$ SNR (record 106m from MITDB) (black line), VFCDM subcomponents: 1 to 12 (red lines).

noise, hence, we want to use the entire $C_{2}(t)$. On the other hand, for a noisy ECG signal, $C_{2}(t)$ is also noisy, consequently, we want to use only the QRS contribution to $C_{2}(t)$. In order to resolve this issue of using either the entire component or only the QRS part of $C_{2}(t)$, we define a power ratio $P_{r}$ as follows: 


$$
P_{r}=\frac{\text { Power of }\left(C_{2}(t) \times m(t)\right)}{\text { Power of }\left(C_{2}(t)\right)} \text {. }
$$

Based on the power ratio we use the following scheme for reconstruction of the signal:

$$
Y_{r}(t)=\begin{array}{rr}
C_{1}(t)+\sum_{i=2}^{4} C_{i}(t) \times m(t), & P_{r}<0.9 \\
C_{1}(t)+C_{2}(t)+\sum_{i=3}^{4} C_{i}(t) \times m(t), & P_{r}>0.9
\end{array}
$$

A higher power ratio means most of the power of $C_{2}(t)$ is concentrated around the QRS complex, so we use the entire $C_{2}(t)$ in the reconstruction. On the other hand, a lower power ratio implies that $C_{2}(t)$ has a substantial amount of power in the masked region, which is the case when $C_{2}(t)$ is noisy. Therefore, we use masked $C_{2}(t)$ when the power ratio is low. Figure 2 shows the denoising steps performed, where the mask itself $(\mathrm{m}(\mathrm{t})$ in panel $\mathrm{d})$ and the masked VFCDM components (in the panels e-g) are plotted in red to make them more distinguishable.

\section{Results}

The performance of the proposed denoising method was validated on the MIT-BIH arrhythmia database and the wearable armband ECG data obtained in our laboratory [26]. The performance of our proposed denoising technique on the MIT database will be compared with three other existing methods, namely NLM [15], wavelet soft thresholding [2], and EMD ASMF [8]. We define three performance metrics: SNR improvement $\left(S N R_{i m p}\right)$, percentage root 


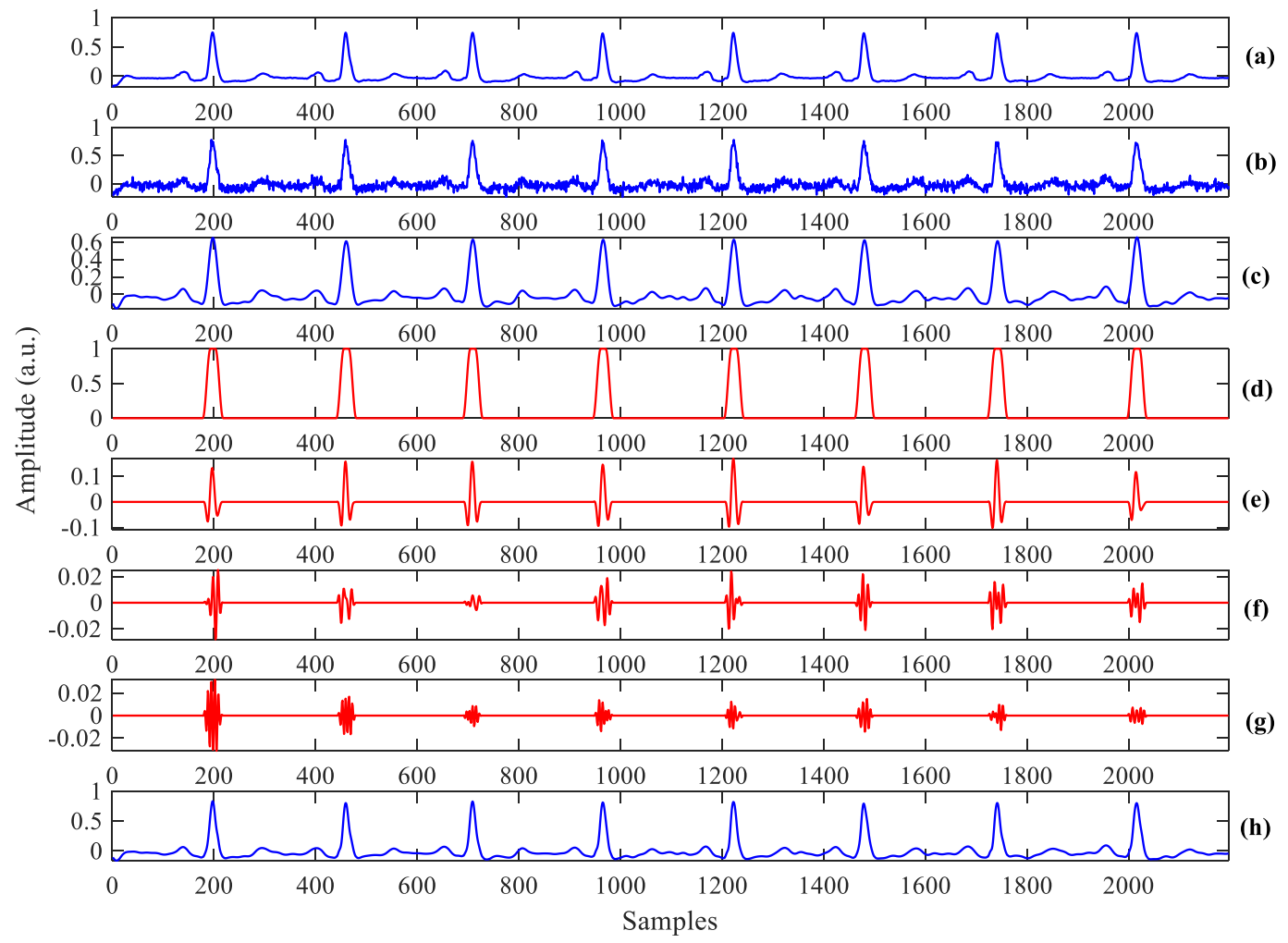

Fig. 2. Denoising steps: (a) original ECG (subject 105) (b) ECG with AWGN noise at $10 \mathrm{~dB}$ SNR (c) first component of VFCDM decomposition $(\mathrm{d})$ mask $(m(t))(\mathrm{e}-\mathrm{g})$ masked VFCDM components 2 to $4(\mathrm{~h})$ final reconstructed signal

mean square difference (PRD), and mean square error (MSE), as these have been used as to evaluate other algorithms' performance [6], [8], [15]. The performance parameters are defined as follows:

$$
\begin{aligned}
& S N R_{i m p}=10 \log _{10} \frac{\sum_{n=1}^{N}\left(Y_{n}[n]-Y[n]\right)^{2}}{\sum_{n=1}^{N}\left(Y_{d}[n]-Y[n]\right)^{2}} \\
& P R D=\sqrt{\frac{\sum_{n=1}^{N}\left(Y_{d}[n]-Y[n]\right)^{2}}{\sum_{n=1}^{N} Y^{2}[n]}} \times 100 \\
& M S E=\frac{1}{N} \sum_{n=1}^{N}\left(Y_{d}[n]-Y[n]\right)^{2}
\end{aligned}
$$

where $Y_{n}[n]$ denotes noisy ECG, $Y[n]$ stands for the original ECG, $Y_{d}[n]$ represents the denoised ECG, and $\mathrm{N}$ is the length of the ECG signal. However, these performance metrics cannot be used to evaluate the denoising 
performance on the armband ECG data since we did not add noise by ourselves, rather, the armband's ECG contains the subject's muscle artifacts.

\subsection{Results on MIT-BIH Arrhythmia Database}

In order to conform with most of the existing denoising methods [6], [8], [15], we considered ECG recordings $100 \mathrm{~m}, 101 \mathrm{~m}, 103 \mathrm{~m}, 105 \mathrm{~m}, 106 \mathrm{~m}, 115 \mathrm{~m}, 215 \mathrm{~m}$, and $230 \mathrm{~m}$ from the MIT database for denoising performance comparison. We simulated different noise sources such as Gaussian white noise, powerline interference, and different colored noises (pink, blue, violet, and red) at 5 different SNR levels (-5 decibels $(\mathrm{dB}), 0 \mathrm{~dB}, 5 \mathrm{~dB}, 10 \mathrm{~dB}, 15 \mathrm{~dB}$, and $20 \mathrm{~dB}$ ). The powerline noise was simulated by producing a sinusoidal signal of $50 \mathrm{~Hz}$ using the approach described in [1] and the colored noise was generated using the method presented in [36].
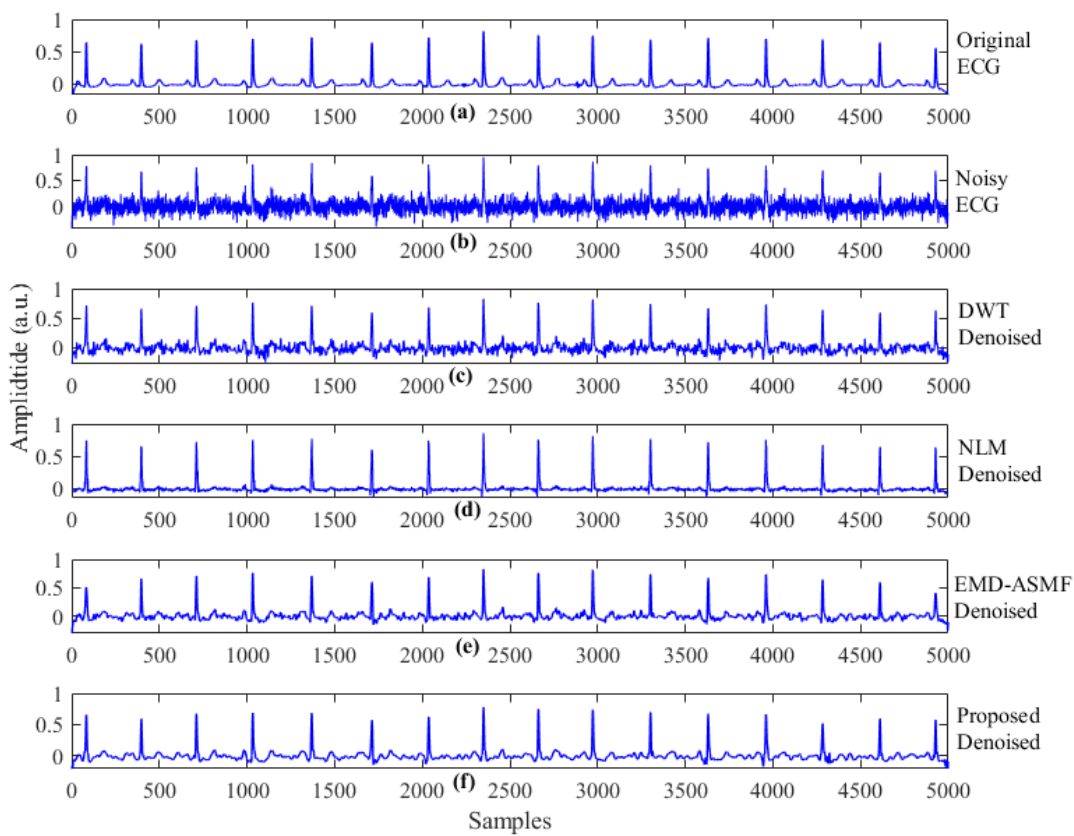

Fig. 3. Blue noise removal in ECG: (a) original signal (record $101 \mathrm{~m}$ from MIT database) (b) ECG with blue noise added at $0 \mathrm{~dB}$ SNR, and denoised ECG using (c) wavelet soft thresholding (d) NLM (e) EMD-ASMF (f) proposed method 


\section{A) Qualitative Analysis}

We evaluate the quality of the denoised signal. Fig. 3 shows the denoised ECG signal using different methods on record $101 \mathrm{~m}$ corrupted with blue noise at $0 \mathrm{~dB}$ SNR. Panel (f) of this figure reveals that the proposed denoising method provides discernible ECG waveforms while preserving ECG morphologies (i.e. P wave, T wave, and QRS complex)

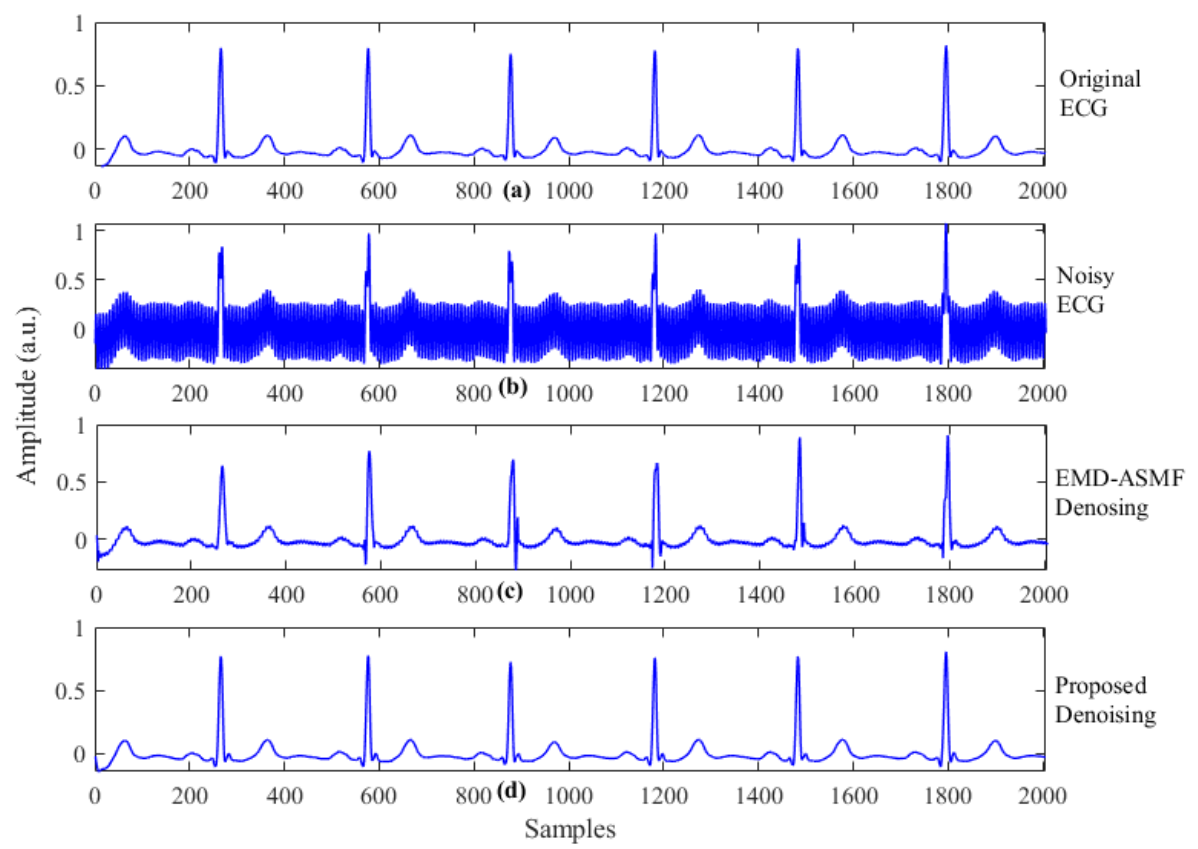

Fig. 4. Power line interference removal: (a) original signal (record 103 from MITDB) (b) ECG with added PLI at -5 dB SNR (c) denoised ECG using EMD-ASMF (d) denoised ECG using the proposed method

even at a low SNR. Note that in some cases, $\mathrm{P}$ and T waves can be of low amplitude. Even in those cases, the lowamplitude waves can be recovered, albeit this depends on the original state of the ECG morphology. A second denoising example is shown in Fig. 4, The ECG record $103 \mathrm{~m}$ was corrupted with power line noise at -5 dB SNR. It can be observed that even at a very low SNR, the proposed method is able to obtain a relatively noise-free ECG segment while the EMD-ASMF method [8] shown in the panel (c) is unable to remove noise, and it distorts the QRS complexes at several time points.

In addition to adding synthetic noise, we also evaluated the proposed method with often-encountered noise artifacts such as baseline wander, muscle noise, and electrode noise, which are all found in the NSTDB. As can be seen from Fig. 5, the proposed method can effectively remove the baseline drift and muscle artifacts even at very low SNR (i.e. $-5 \mathrm{~dB}$ ). The performance of the method even on electrode noise is also reasonably good, given that electrode noise is considered to be one of the most difficult to remove. 
The efficacy of the proposed method on ECG segments with arrhythmia is also visible from Fig. 5, which shows premature ventricular contraction (PVC) with bigeminy and trigemini patterns, and atrial flutter/fibrillation. All ECG records with these arrhythmias were corrupted by AWGN at $0 \mathrm{~dB}$ SNR and then the proposed denoising was applied.

As shown in Fig. 5, in each case the denoised and original ECG (prior to AWGN contamination) nearly overlap, which illustrates that the proposed denoising technique is also effective even for ECGs with arrhythmias.
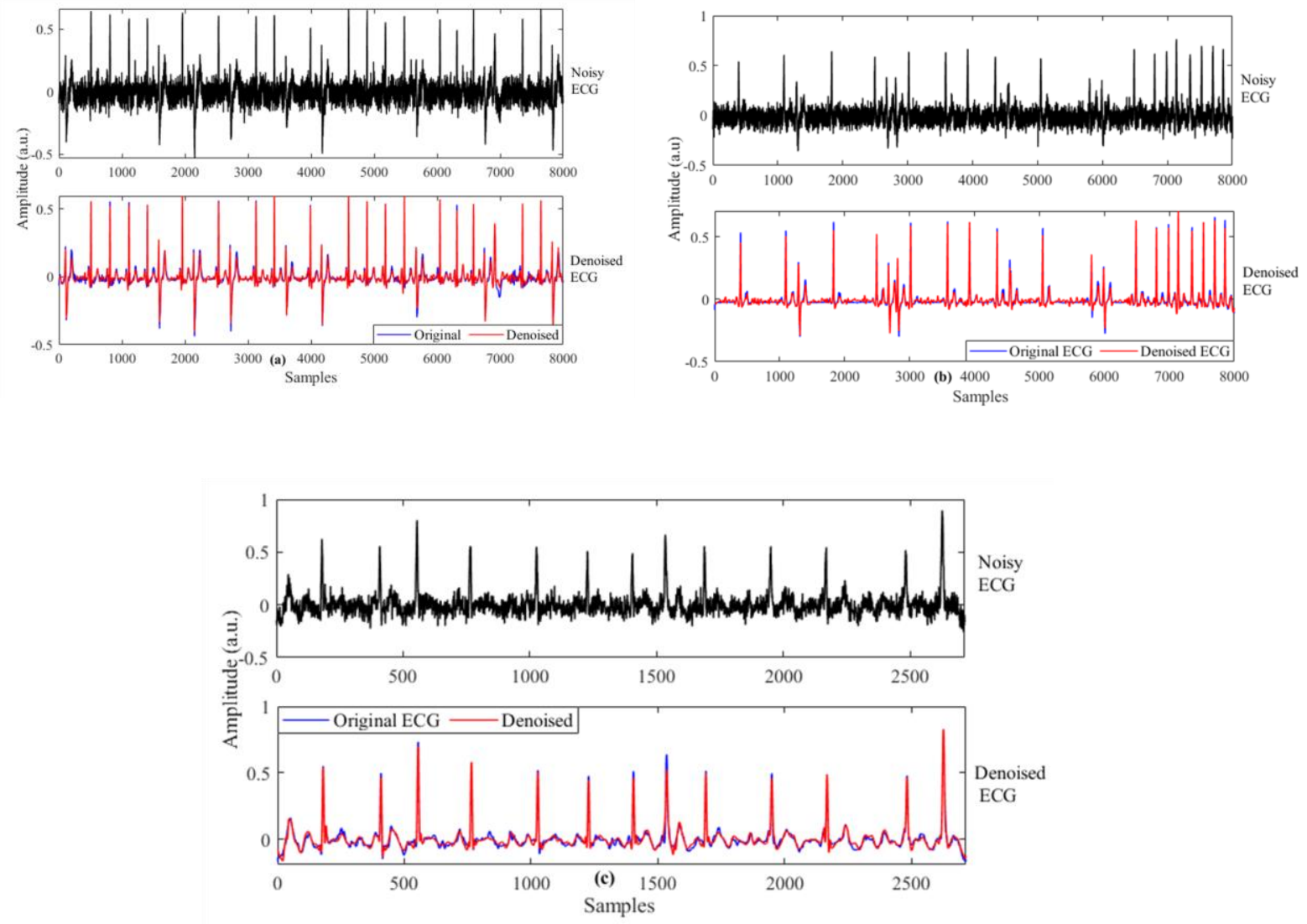

Fig. 5. Proposed denoising applied on arrhythmic ECGs: (a) PVCs with bigeminy (record 106 from MITDB) (b) PVC with ventricular trigeminy (record 201 from MITDB) (c) Atrial Flutter/fibrillation (record 222 from MITDB) 


\section{B) Quantitative Analysis}

For quantitative analysis, we used the performance metrics defined in Eqs. (7-9). At a particular SNR level, a better denoising method is expected to provide higher $S N R_{\text {imp }}$, and lower PRD and MSE.

Fig. 6 shows the $S N R_{i m p}$ for all methods, at different SNR levels of added GWN, for the ECG records considered. It can be observed that the proposed denoising technique provided better $S N R_{i m p}$ at almost all SNR levels. Figs. 7 and 8 present a comparison of PRD and MSE results, respectively, for different denoising methods when GWN was added. These plots indicate that the proposed denoising method provides lower PRD and MSE than do the other methods considered.

As mentioned earlier, the proposed method performed well in removing power line interference. This is also visible from the performance metrics as well. Figs. 9 and 10 show $S N R_{\text {imp }}$ and PRD results, respectively, using different denoising methods at different levels of power line noise. These figures show that the proposed denoising method has 


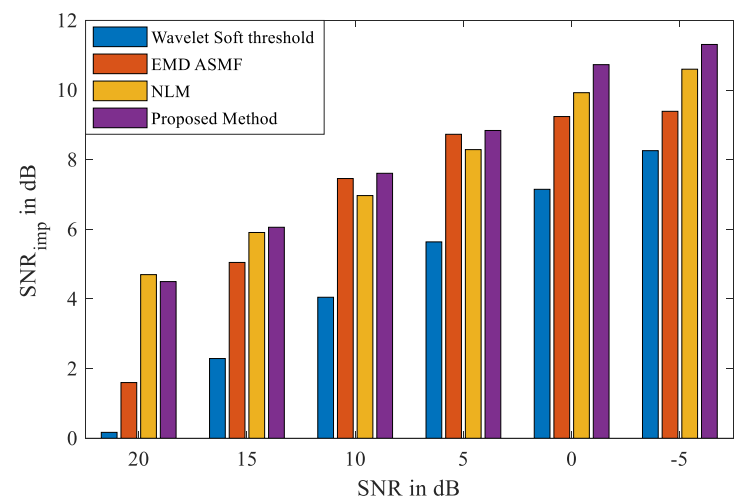

Fig. 6. Comparison of $S N R_{\text {imp }}$ for different denoising methods with Gaussian white noise contamination.

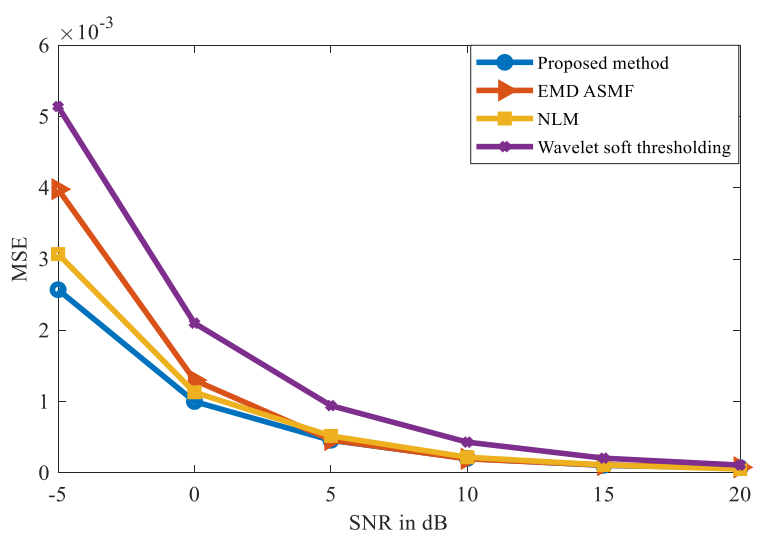

Fig. 8. Comparison of $M S E$ for different denoising methods with Gaussian white noise.

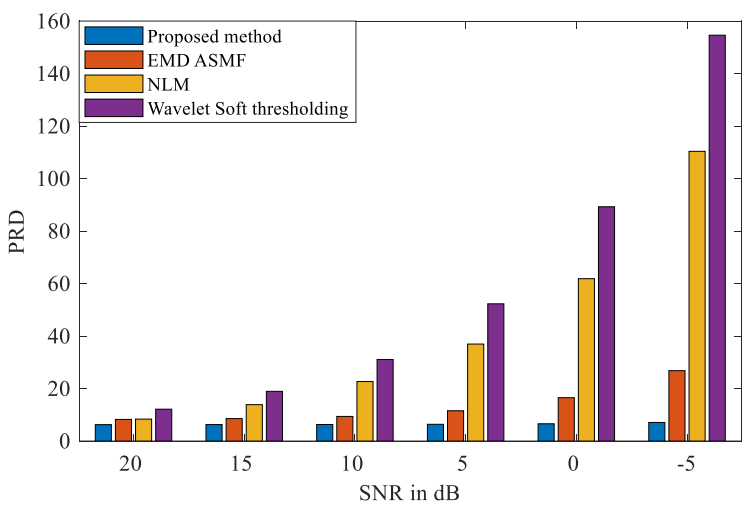

Fig. 10. Comparison of $P R D$ with different denoising methods for removal of power line interference.

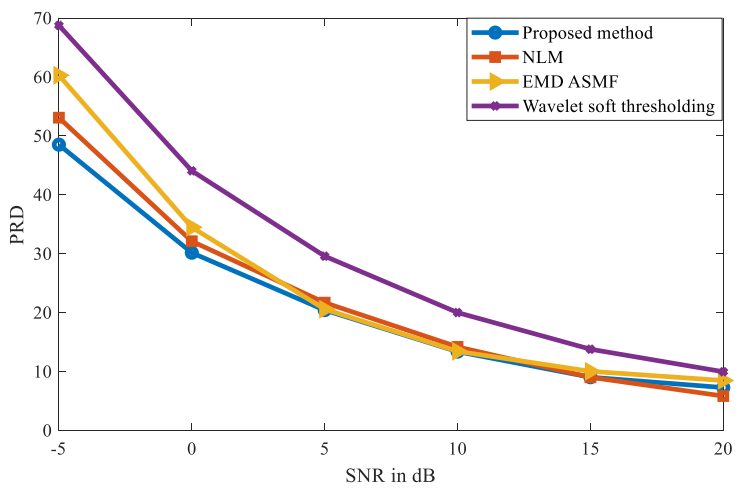

Fig. 7. Comparison of $P R D$ for different denoising methods with Gaussian white noise contamination.

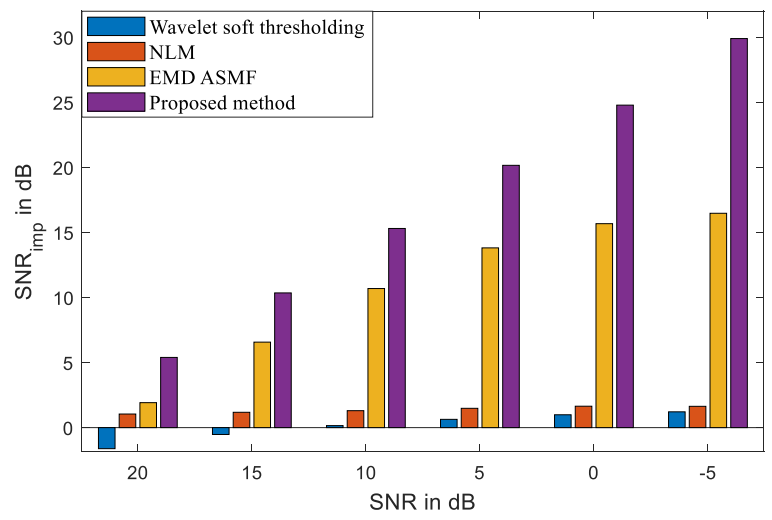

Fig. 9. Comparison of $S N R_{\text {imp }}$ with different denoising methods for removal of power line interference.

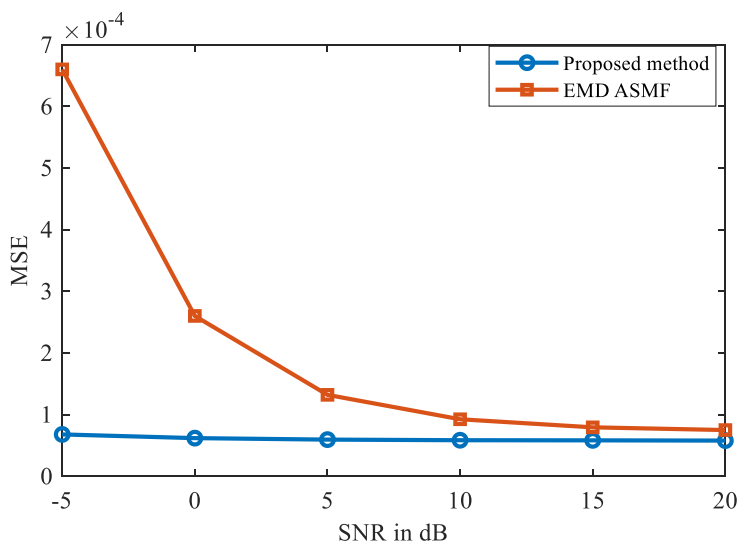

Fig. 11. Comparison of $M S E$ of different denoising methods for power line interference.

significantly higher $S N R_{\text {imp }}$ and lower PRD when compared with the other denoising techniques. Fig. 11. shows a comparison of MSE results using different denoising methods for different levels of power line noise. It should be 
noted that the figure compares only two methods (the proposed and EMD-ASMF). The other methods were not used because of their high MSE values.

TABLE I

SNR IMPROVEMENT USING THREE DIFFERENT TYPES OF ADDITIVE COLORED NOISE

\begin{tabular}{|c|c|c|c|c|c|c|}
\hline \multicolumn{7}{|c|}{ SNR in DB } \\
\hline \multirow[t]{2}{*}{ Methods } & \multicolumn{5}{|c|}{ Blue Noise } & \multirow[b]{2}{*}{-5} \\
\hline & 20 & 15 & 10 & 5 & 0 & \\
\hline Proposed work & 5.0944 & 9.3574 & 12.7024 & 14.8720 & 16.4165 & 18.4695 \\
\hline EMD-ASMF & 2.3103 & 6.7723 & 10.9340 & 14.2621 & 16.3226 & 17.2903 \\
\hline NLM & 4.6744 & 5.8900 & 6.8032 & 7.8960 & 9.3034 & 10.3026 \\
\hline DWT & 2.1952 & 5.0942 & 7.5028 & 9.6625 & 11.8605 & 14.2818 \\
\hline \multicolumn{7}{|c|}{ Pink Noise } \\
\hline Proposed work & 0.4829 & 0.9825 & 3.4747 & 5.2371 & 6.4866 & 7.6391 \\
\hline EMD-ASMF & -1.0678 & 0.4826 & 1.1685 & 1.4352 & 1.5253 & 1.5213 \\
\hline NLM & 1.5708 & 2.1777 & 2.9078 & 3.6712 & 4.2021 & 4.1623 \\
\hline DWT & -1.6188 & -0.5260 & 0.1608 & 0.6376 & 0.9905 & 1.2164 \\
\hline \multicolumn{7}{|c|}{ Violet Noise } \\
\hline Proposed work & 5.4434 & 10.2839 & 14.8359 & 18.7309 & 21.5255 & 23.2784 \\
\hline EMD-ASMF & 2.4810 & 7.1487 & 11.8377 & 16.3480 & 20.1929 & 22.8851 \\
\hline NLM & 4.4247 & 5.5835 & 6.4479 & 7.4529 & 8.7023 & 9.5548 \\
\hline$\overline{\text { DWT }}$ & 3.5503 & 7.1352 & 10.2412 & 12.8445 & 15.1321 & 17.6662 \\
\hline
\end{tabular}

TABLE II

PRD USING THREE DIFFERENT TYPES OF ADDITIVE COLORED NOISE

\begin{tabular}{|c|c|c|c|c|c|c|}
\hline \multicolumn{7}{|c|}{ SNR in $\mathrm{dB}$} \\
\hline \multirow[t]{2}{*}{ Methods } & \multicolumn{6}{|c|}{ Blue noise } \\
\hline & 20 & 15 & 10 & 5 & 0 & -5 \\
\hline Proposed work & 6.3162 & 6.6447 & 7.6078 & 10.0169 & 15.2195 & 20.9380 \\
\hline EMD-ASMF & 7.8622 & 8.3507 & 9.1443 & 10.9798 & 15.3075 & 24.3119 \\
\hline NLM & 6.1321 & 9.0661 & 14.4856 & 22.7010 & 34.4293 & 55.1862 \\
\hline DWT & 8.0125 & 10.0851 & 13.5351 & 18.8132 & 25.9923 & 34.8458 \\
\hline \multicolumn{7}{|c|}{ Pink Noise } \\
\hline Proposed work & 10.8961 & 16.3299 & 21.4156 & 30.6806 & 47.5306 & 74.1099 \\
\hline EMD-ASMF & 11.8464 & 17.2420 & 27.6528 & 47.9171 & 84.0416 & 149.2678 \\
\hline NLM & 9.3620 & 14.2122 & 22.6752 & 36.9512 & 61.8522 & 110.3761 \\
\hline DWT & 12.1424 & 18.9401 & 31.0743 & 52.2812 & 89.2512 & 154.6188 \\
\hline \multicolumn{7}{|c|}{ Violet Noise } \\
\hline Proposed work & 6.1790 & 6.2344 & 6.4198 & 6.9632 & 8.4366 & 11.8962 \\
\hline EMD-ASMF & 7.7177 & 8.0254 & 8.3177 & 8.7559 & 9.9083 & 12.8178 \\
\hline NLM & 6.0433 & 9.3884 & 15.0879 & 23.8854 & 36.8617 & 59.9528 \\
\hline DWT & 6.9331 & 8.0521 & 9.9215 & 13.0995 & 17.8841 & 23.9432 \\
\hline
\end{tabular}


TABLE III

MSE $\left(\times 10^{-3}\right)$ ON THREE DIFFERENT ADDITIVE COLORED NOISE TYPES

\begin{tabular}{|c|c|c|c|c|c|c|}
\hline \multicolumn{7}{|c|}{$\mathrm{SNR}$ in $\mathrm{dB}$} \\
\hline \multirow[t]{2}{*}{ Methods } & \multicolumn{6}{|c|}{ Blue Noise } \\
\hline & 20 & 15 & 10 & 5 & 0 & -5 \\
\hline Proposed work & 0.058 & 0.063 & 0.079 & 0.130 & 0.259 & 0.508 \\
\hline EMD-ASMF & 0.067 & 0.075 & 0.089 & 0.129 & 0.253 & 0.643 \\
\hline NLM & 0.047 & 0.089 & 0.227 & 0.566 & 1.290 & 3.310 \\
\hline DWT & 0.071 & 0.110 & 0.197 & 0.381 & 0.731 & 1.310 \\
\hline \multicolumn{7}{|c|}{ Pink Noise } \\
\hline Proposed work & 0.137 & 0.291 & 0.497 & 1.040 & 2.460 & 5.880 \\
\hline EMD-ASMF & 0.139 & 0.307 & 0.834 & 2.490 & 7.720 & 24.500 \\
\hline NLM & 0.095 & 0.278 & 0.566 & 1.500 & 4.200 & 13.400 \\
\hline DWT & 0.16 & 0.389 & 1.050 & 2.980 & 8.700 & 26.200 \\
\hline \multicolumn{7}{|c|}{ Violet Noise } \\
\hline Proposed work & 0.054 & 0.057 & 0.060 & 0.068 & 0.096 & 0.177 \\
\hline EMD-ASMF & 0.064 & 0.069 & 0.075 & 0.083 & 0.105 & 0.176 \\
\hline NLM & 0.040 & 0.095 & 0.247 & 0.626 & 1.480 & 3.900 \\
\hline DWT & 0.055 & 0.072 & 0.107 & 0.185 & 0.344 & 0.623 \\
\hline
\end{tabular}

Finally, we considered three different colored noise scenarios, namely blue, pink, and violet to contaminate the ECG signals at different SNR levels and evaluated the denoising performance of the all methods considered. The detailed results are shown in Tables I, II, and III, respectively. Table I presents the $S N R_{i m p}$ for all the denoising techniques, at different levels of colored noise. It can be observed from Table I that for blue and violet noise, most of the denoising techniques performed well and provided higher $S N R_{\text {imp }}$. The proposed method has the highest SNR improvement at almost all SNR levels. In the case of pink noise, most of the methods failed to provide good denoising performance, which can be observed from the $S N R_{\text {imp }}$ values. The table shows that even in the case of pink noise, the proposed method provided reasonably higher $S N R_{i m p}$. The same conclusion can be drawn from Tables II and III, which show the PRD and MSE values, respectively. The proposed method provided significantly lower PRD and MSE values when compared with the other existing techniques.

In addition to $S N R_{i m p}, P R D$, and $M S E$ as performance metrics, we have also used the wavelet energy diagnostic distortions (WEDD) metric, as proposed in [37]. WEDD is a standard and popular diagnostic distortion measure that has been used previously in a recent ECG denoising literature[38]. The lower the WEDD, the better the denoising performance. According to the criteria given in [37], the denoising performance can be ranked as (1) excellent (WEDD < 4.517\%), (2) very good ( WEDD is within 4.517\% - 6.914\%), (3) good (WEDD is within 6.914\% - 11.125\% ), (4) not bad (WEDD is within 11.125\% - 13.56\%), (5) Bad (WEDD > 13.56\%),). 
Table IV shows the denoising performance comparison in terms of WEDD at different levels of SNR. It can be seen from the table that the proposed VFCDM-based denoising results in smaller WEDD in most of the SNR cases (especially at higher SNR). At $20 \mathrm{~dB}$, the NLM has slightly lower WEDD than the proposed denoising method. However, this smaller WEDD is insignificant since the signal at $20 \mathrm{~dB}$ is not considered to be noisy. The proposed denoising method was able to remove the power line interference noise even at a very low SNR level (e.g. $<0 \mathrm{~dB})$. In terms of WEDD, the proposed denoising method performs excellently at $20 \mathrm{~dB}$, very good at $15 \mathrm{~dB}, 10 \mathrm{~dB}, 5 \mathrm{~dB}$, and $0 \mathrm{~dB}$, and good at $-5 \mathrm{~dB}$, according to the defined criteria [37].

Finally, we analyzed the performance of the proposed denoising method on MIT-BIH's NSTDB. This database contains three different types of noise sources commonly encountered in practice, namely, baseline wander (BW), muscle noise (MN), and electrode motion artefact (EM) [27]. We added these three noise sources at 6 different SNR levels to the ECG records from the MITDB. The performance of the proposed denoising method was evaluated in terms of both non-diagnostic distortion $\left(S N R_{\text {imp }}, P R D\right)$ and diagnostic distortion (WEDD). The proposed method's performance is shown in Table $\mathrm{V}$. As shown, the proposed method was able to remove the baseline wander even at very low SNR, providing higher $S N R_{\text {imp }}$, and lower PRD and WEDD values. The worst WEDD is $9.05 \%$ (at $-5 \mathrm{~dB}$ ) which still falls in the "good" range (6.914\% - 11.125\%). The proposed denoising method showed promising results in removing muscle noise as well. It should be noted that many of the previous studies [8], [39] simulated muscle noise using random noise generation, however, in this study, we used true muscle noise data. Removing electrode noise is considered the most troublesome since it can mimic the appearance of ectopic beats and the noise frequency components' dynamics significantly overlap with the ECG components [10]. The proposed denoising technique performed satisfactorily in removing electrode noise, as also shown in Table V.

TABLE IV

DIAGNOSTIC DISTORTION COMPARISON (WEDD)

\begin{tabular}{|c|c|c|c|c|c|c|}
\hline \multicolumn{7}{|c|}{ SNR in $\mathrm{dB}$} \\
\hline \multirow[t]{2}{*}{ Methods } & \multicolumn{6}{|c|}{ AWGN } \\
\hline & 20 & 15 & 10 & 5 & 0 & -5 \\
\hline Proposed work & $5.01 \%$ & $6.79 \%$ & $11.01 \%$ & $15.28 \%$ & $22.41 \%$ & $43.21 \%$ \\
\hline EMD-ASMF & $6.87 \%$ & $8.63 \%$ & $12.34 \%$ & $19.64 \%$ & $33.27 \%$ & $58.51 \%$ \\
\hline NLM & $4.68 \%$ & $6.90 \%$ & $11.21 \%$ & $17.27 \%$ & $25.74 \%$ & $47.99 \%$ \\
\hline DWT & $8.51 \%$ & $10.79 \%$ & $18.08 \%$ & $24.58 \%$ & $38.79 \%$ & $59.68 \%$ \\
\hline \multicolumn{7}{|c|}{ Power line interference (PLI) } \\
\hline Proposed work & $4.13 \%$ & $5.76 \%$ & $5.78 \%$ & $5.90 \%$ & $6.28 \%$ & $7.31 \%$ \\
\hline EMD-ASMF & $5.85 \%$ & $6.00 \%$ & $6.44 \%$ & $7.57 \%$ & $10.17 \%$ & $15.57 \%$ \\
\hline
\end{tabular}


TABLE V

PERFORMANCE ON NSTDB

\begin{tabular}{|c|c|c|c|c|c|c|}
\hline \multicolumn{7}{|c|}{ SNR in $\mathrm{dB}$} \\
\hline \multirow[t]{2}{*}{ Parameter } & \multicolumn{6}{|c|}{ Baseline wander } \\
\hline & 20 & 15 & 10 & 5 & 0 & -5 \\
\hline$S N R \_i m p$ & 3.8918 & 7.8178 & 12.4083 & 16.5442 & 20.0098 & 22.44 \\
\hline PRD & $5.04 \%$ & $6.77 \%$ & $7.58 \%$ & $8.37 \%$ & $9.99 \%$ & $11.43 \%$ \\
\hline WEDD & $4.81 \%$ & $5.01 \%$ & $6.31 \%$ & $6.83 \%$ & $7.13 \%$ & $9.05 \%$ \\
\hline \multicolumn{7}{|c|}{ Muscle Noise } \\
\hline SNR_imp & 4.1701 & 7.5015 & 9.7676 & 10.8946 & 12.5031 & 13.9935 \\
\hline PRD & $5.79 \%$ & $7.44 \%$ & $10.93 \%$ & $15.71 \%$ & $23.82 \%$ & $41.05 \%$ \\
\hline WEDD & $4.82 \%$ & $6.52 \%$ & $8.94 \%$ & $13.72 \%$ & $20.32 \%$ & $35.67 \%$ \\
\hline \multicolumn{7}{|c|}{ Electrode Noise } \\
\hline SNR_imp & 3.8092 & 6.9039 & 7.8119 & 8.8452 & 9.3541 & 9.9778 \\
\hline PRD & $7.01 \%$ & $9.81 \%$ & $12.01 \%$ & $21.09 \%$ & $32.57 \%$ & $56.91 \%$ \\
\hline WEDD & $5.36 \%$ & $8.61 \%$ & $11.07 \%$ & $19.85 \%$ & $29.71 \%$ & $48.67 \%$ \\
\hline
\end{tabular}

The overall results suggest that the proposed method has superior denoising performance in almost all noisy conditions. The NLM [15] method is quite effective in removing GWN but it does not work well on other noise types such as PLI and colored noise. On the other hand, the EMD-ASMF [8] works well in removing power line noise, but its performance is poor with pink noise. DWT-based denoising [2] could not fully remove the noise when the ECG signal was highly corrupted. Note that all of these compared methods performed poorly on NSTDB noise, which is why their results were not included in Table V.

\subsection{Results on the Armband ECG Data}

In order to evaluate the performance of the proposed denoising technique on the armband ECG, we randomly chose 40 ten-second segments of both noisy and clean ECG channels from the armband ECG database [26]. Adjudication of clean versus noisy ECG data was determined visually by three of the authors (N.R., K.H.C., and J.L.) based on the presence of $\mathrm{P}, \mathrm{Q}, \mathrm{R}, \mathrm{S}$, and $\mathrm{T}$ waves in the ECG segments. The denoising performance on the ECG segments was evaluated and compared in terms of accurate R peak detection and SNR improvement. For R peak detection, we used the Pan and Tompkins R-peak detection algorithm [40] on the denoised sequences. Detected R peaks were visually inspected by three independent experts and the majority vote was taken as the correct decision. SNR improvement was calculated by subtracting the noisy signal SNR from the denoised signal SNR, as described in [23].

QRS complex detection on the representative segments of noisy armband and denoised armband ECG signals is shown in Fig. 12. It can be observed that due to excessive EMG noise artifacts, there are several R peak misdetections 
and false positives in the noisy armband data (upper panel), which are avoided in the denoised armband ECG (bottom panel). The summary of the denoising performance and comparison on the armband ECG data is provided in Table VI. As we can see from the table, the proposed denoising method provides the highest percentage (92.9577\%) of correctly detected R peaks. EMD-ASMF provides nearly the same percentage of correctly detected R peaks. However, this method requires an external QRS complex detector in order to preserve them in the process of denoising, and hence, the performance of EMD-ASMF is quite dependent on QRS complex detection algorithm. Table VI also shows that the proposed denoising method provides significantly higher SNR improvement (1.4595) when compared to that of the DWT and EMD-ASMF denoising methods. As stated earlier, the NLM has a parameter bandwidth which is dependent on the noise standard deviation, which is unknown in the noisy ECG data. Therefore, we could not compare the proposed denoising method with NLM for the armband ECG data.

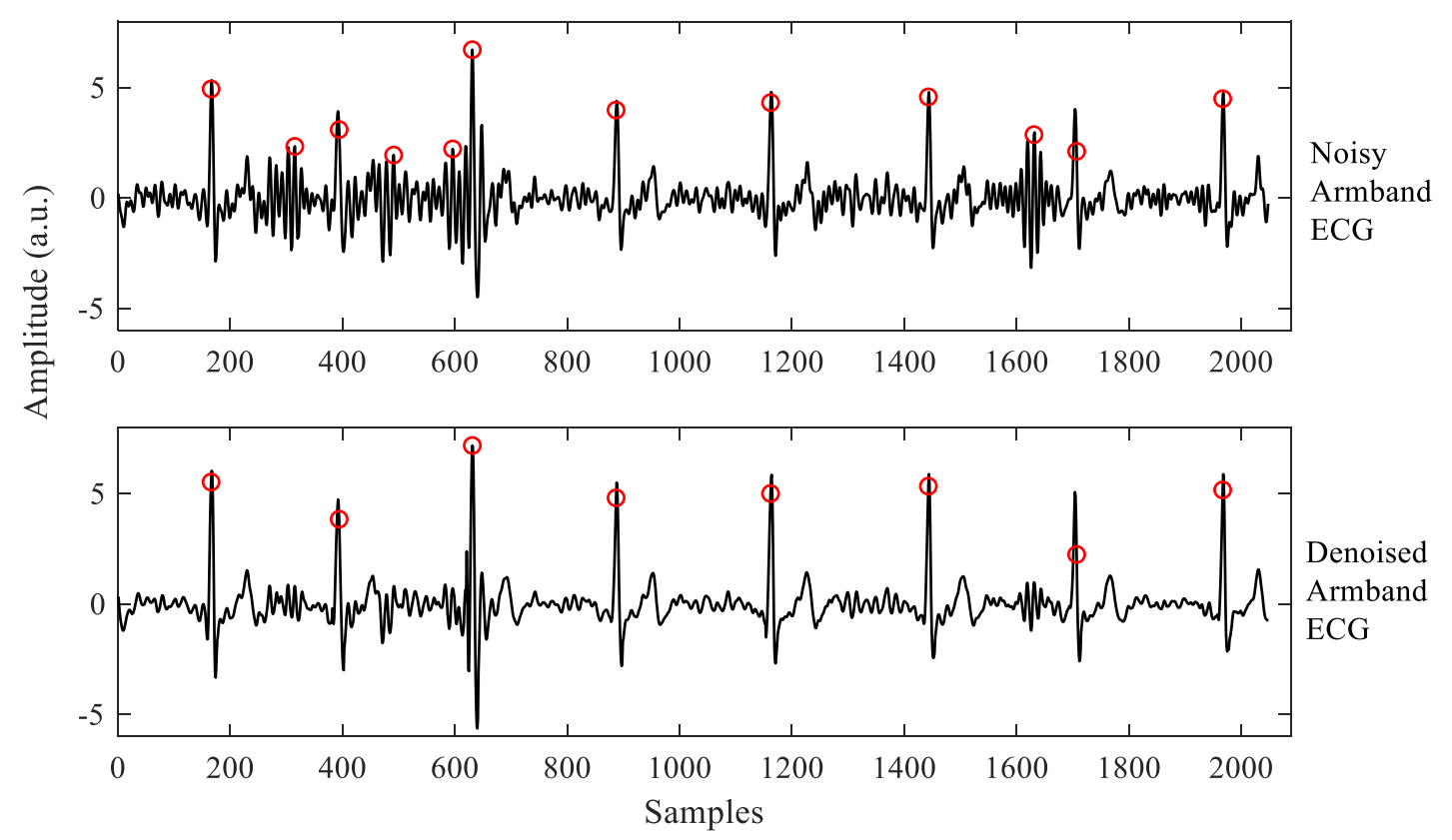

Figure 12. QRS complex detection on (a) armband ECG segment with muscle artifact (b) the same segment, denoised.

TABLE VI

QRS DETECTION PERFORMANCE COMPARISON

\begin{tabular}{|c|c|c|}
\hline Denoising Method & Correctly detected R peaks & SNR $_{\text {imp }}($ mean \pm sd $)$ \\
\hline DWT & $79.4871 \%$ & $0.0569 \pm 0.0376$ \\
\hline EMD-ASMF & $92.8826 \%$ & $1.0568 \pm 0.6342$ \\
\hline Proposed method & $92.9577 \%$ & $1.4595 \pm 0.6326$ \\
\hline
\end{tabular}




\section{Discussion}

The results presented in this paper demonstrate that the proposed denoising method provided better denoising performance when compared to other denoising techniques in a variety of noise-corrupted scenarios. We showed that the VFCDM-based subband decomposition of noisy ECG signals was more effective than EMD or wavelet-based denoising techniques in separating noisy components from the clean ECG. The proposed denoising method provided cleaner ECG segments and also retained ECG morphologies (i.e. P wave, T wave, and QRS complexes).

We considered ECG signals corrupted with different types of noise at different levels of SNR to evaluate the denoising performance on a variety of noisy conditions. The results in this paper indicate that most of the existing methods considered in this paper did not perform adequately in removing noise from a wide range of sources. For example, NLM [15] provided good denoising performance in AWGN noise but its performance on removing PLI and colored noise was found to be poor. The EMD-ASMF [8] technique provided poor denoising performance on ECG signals corrupted with pink colored noise while the wavelet soft thresholding method [2] could not remove noise entirely in most of the cases.

The qualitative and quantitative analysis of the denoising results indicate that the proposed denoising technique can provide better-quality denoised ECG with higher $\mathrm{SNR}_{\text {imp }}$ as well as lower PRD and MSE values than the existing denoising techniques considered. In ECGs contaminated with AWGN, the proposed denoising method provided higher SNR_imp, and lower PRD and MSE at almost all SNR levels than NLM, EMD-ASMF and DWT-based techniques. At $20 \mathrm{~dB}$ SNR, the SNR improvement using the NLM method [15] was slightly higher than our proposed method, however, this improvement was not significant because it had negligible effect on the ECG quality. The same was true for PRD and MSE as well.

The denoising performance of our proposed method on the PLI-corrupted ECG signals was found to be better than any of the methods compared. The sub-band decomposition of the noisy signals enabled the noise-free reconstruction of the ECG at a low SNR level. The $\mathrm{SNR}_{\mathrm{imp}}$ resulting from the proposed denoising method was significantly larger than that of the existing methods. Moreover, the PRD and MSE values were almost constant across different levels of SNR, which indicates that the proposed method can remove PLI noise almost equally good at different levels of SNR. 
Even though the EMD-ASMF method can remove PLI to a great extent, this method failed to retain the QRS amplitudes, especially at lower input SNR levels. Moreover, it was not able to remove noise components, especially at low SNR levels.

Similar to the AWGN and PLI cases, the proposed method also showed superior denoising performance on ECGs corrupted with colored noise, when compared to the existing denoising techniques. While the other methods showed low $\mathrm{SNR}_{\mathrm{imp}}$ and higher PRD and MSE values for the ECGs contaminated with pink noise at low input SNR levels, in this case the proposed method provided sufficient $\mathrm{SNR}_{\mathrm{imp}}$, and reasonable PRD and MSE values.

The major advantage of the proposed method is that it has been tested using different noise sources that are often observed in practice, such as baseline wander, muscle noise, and electrode noise, whereas most of the existing denoising methods were evaluated using synthetic noise. The proposed method was shown to remove baseline wander even at very low SNR levels without any significant distortion of the ECG morphologies (WEDD is a maximum of $9.05 \%$ at $-5 \mathrm{~dB}$ SNR). Sufficiently good results were obtained in the case of muscle noise as well. Moreover, the application of the proposed denoising algorithm on the wearable armband ECG data which are contaminated with muscle artifacts showed significantly better performance when compared to the other methods considered in this study. The proposed method showed a moderate performance in the case of electrode noise situations. This is because electrode noise can mimic the appearance of ectopic beats and the dynamics of the noise are highly overlapped with the ECG components. Thus, removing electrode noise is known to be difficult.

Finally, the application of the proposed denoising method on the noisy armband ECG data did improve the QRS complex detection accuracy. This result indicates that the proposed denoising method can significantly enhance the accuracy of R-R interval based cardiac arrhythmia (e.g. atrial fibrillation (AF) [41]) detection.

\section{Conclusions}

We presented a novel ECG denoising technique using a high time-frequency resolution method. The proposed method was validated on the standard MIT-BIH arrhythmia database with a variety of noise (i.e. AWGN, colored noise (blue, pink, and violet), PLI, baseline wander, electrode noise, and muscle artifacts) at different SNR levels, and its performance was compared with three other existing denoising methods. In most of the noisy scenarios, the 
proposed method showed better denoising performance over the other methods. The application on the arrhythmic ECGs suggest that the proposed denoising method is equally applicable for both regular and arrhythmic ECGs.

Finally, the proposed method was successfully applied to the armband ECG signals and it was able to remove significant EMG artifacts, consequently providing more accurate R-peak detection. The QRS complex detection results on the denoised armband data demonstrate that the proposed denoising method could significantly enhance the potential of the armband device to be used for continuous monitoring of AF, and without the skin irritation that has been a problem for Holter monitors because of their hydrogel electrodes.

\section{Conflicts of Interest}

The authors declare no conflicts of interest.

\section{Acknowledgement}

This project has received funding from the European Union's Framework Programme for Research and Innovation Horizon 2020 (2014-2020) under the Marie Skłodowska-Curie Grant Agreement No. 745755. This work was also supported by the Government of Aragón and the European Social Fund (EU) through the BSICoS group (T96), and by CIBER in Bioengineering, Biomaterials \& Nanomedicine (CIBER-BBN) through Instituto de Salud Carlos III. The computation was performed by the ICTS NANBIOSIS, specifically by the High-Performance Computing Unit of CIBER-BBN at University of Zaragoza. This work was also supported by NSF SBIR Phase I (\#1746589) and R43 HL135961.

\section{References}

[1] W. Jenkal, R. Latif, A. Toumanari, A. Dliou, O. El B'charri, and F. M. R. Maoulainine, "An efficient algorithm of ECG signal denoising using the adaptive dual threshold filter and the discrete wavelet transform," Biocybern. Biomed. Eng., vol. 36, no. 3, pp. 499-508, Jan. 2016, doi: 10.1016/j.bbe.2016.04.001.

[2] B. N. Singh and A. K. Tiwari, "Optimal selection of wavelet basis function applied to ECG signal denoising," Digit. Signal Process., vol. 16, no. 3, pp. 275-287, May 2006, doi: 10.1016/j.dsp.2005.12.003.

[3] Md. A. Awal, S. S. Mostafa, M. Ahmad, and M. A. Rashid, "An adaptive level dependent wavelet thresholding for ECG denoising," Biocybern. Biomed. Eng., vol. 34, no. 4, pp. 238-249, Jan. 2014, doi: 10.1016/j.bbe.2014.03.002.

[4] G. Lu et al., "Removing ECG noise from surface EMG signals using adaptive filtering," Neurosci. Lett., vol. 462, no. 1, pp. 14-19, Sep. 2009, doi: 10.1016/j.neulet.2009.06.063.

[5] C. Marque, C. Bisch, R. Dantas, S. Elayoubi, V. Brosse, and C. Pérot, "Adaptive filtering for ECG rejection from surface EMG recordings," J. Electromyogr. Kinesiol., vol. 15, no. 3, pp. 310-315, Jun. 2005, doi: 10.1016/j.jelekin.2004.10.001.

[6] Md. A. Kabir and C. Shahnaz, "Denoising of ECG signals based on noise reduction algorithms in EMD and wavelet domains," Biomed. Signal Process. Control, vol. 7, no. 5, pp. 481-489, Sep. 2012, doi: 10.1016/j.bspc.2011.11.003.

[7] M. Blanco-Velasco, B. Weng, and K. E. Barner, "ECG signal denoising and baseline wander correction based on the empirical mode decomposition," Comput. Biol. Med., vol. 38, no. 1, pp. 1-13, Jan. 2008, doi: 10.1016/j.compbiomed.2007.06.003.

[8] M. Rakshit and S. Das, "An efficient ECG denoising methodology using empirical mode decomposition and adaptive switching mean filter," Biomed. Signal Process. Control, vol. 40, pp. 140-148, Feb. 2018, doi: 10.1016/j.bspc.2017.09.020.

[9] P. Nguyen and J.-M. Kim, "Adaptive ECG denoising using genetic algorithm-based thresholding and ensemble empirical mode decomposition,” Inf. Sci. Int. J., vol. 373, no. C, pp. 499-511, Dec. 2016, doi: 10.1016/j.ins.2016.09.033.

[10] V. X. Afonso, W. J. Tompkins, T. Q. Nguyen, K. Michler, and Shen Luo, "Comparing stress ECG enhancement algorithms," IEEE Eng. Med. Biol. Mag., vol. 15, no. 3, pp. 37-44, May 1996, doi: 10.1109/51.499756.

[11] I. I. Christov and I. K. Daskalov, "Filtering of electromyogram artifacts from the electrocardiogram," Med. Eng. Phys., vol. 21, no. 10, pp. 731-736, Dec. 1999, doi: 10.1016/S1350-4533(99)00098-3. 
[12] R. Sameni, M. B. Shamsollahi, C. Jutten, and G. D. Clifford, "A Nonlinear Bayesian Filtering Framework for ECG Denoising,” IEEE Trans. Biomed. Eng., vol. 54, no. 12, pp. 2172-2185, Dec. 2007, doi: 10.1109/TBME.2007.897817.

[13] E. Gokgoz and A. Subasi, "Effect of multiscale PCA de-noising on EMG signal classification for diagnosis of neuromuscular disorders," J. Med. Syst., vol. 38, no. 4, p. 31, Apr. 2014, doi: 10.1007/s10916-014-0031-3.

[14] T. He, G. Clifford, and L. Tarassenko, "Application of independent component analysis in removing artefacts from the electrocardiogram," Neural Comput. Appl., vol. 15, no. 2, pp. 105-116, Apr. 2006, doi: 10.1007/s00521-005-0013-y.

[15] B. H. Tracey and E. L. Miller, "Nonlocal Means Denoising of ECG Signals," IEEE Trans. Biomed. Eng., vol. 59, no. 9, pp. 2383-2386, Sep. 2012, doi: 10.1109/TBME.2012.2208964.

[16] İ. Güler and E. D. Übeylı", "ECG beat classifier designed by combined neural network model," Pattern Recognit., vol. 38, no. 2, pp. 199208, Feb. 2005, doi: 10.1016/j.patcog.2004.06.009.

[17] H.-T. Chiang, Y.-Y. Hsieh, S.-W. Fu, K.-H. Hung, Y. Tsao, and S.-Y. Chien, "Noise Reduction in ECG Signals Using Fully Convolutional Denoising Autoencoders," IEEE Access, vol. 7, pp. 60806-60813, 2019, doi: 10.1109/ACCESS.2019.2912036.

[18] N. E. Huang et al., "The empirical mode decomposition and the Hilbert spectrum for nonlinear and non-stationary time series analysis," Proc. R. Soc. Lond. Ser. Math. Phys. Eng. Sci., vol. 454, no. 1971, pp. 903-995, Mar. 1998, doi: 10.1098/rspa.1998.0193.

[19] P. Singh and G. Pradhan, "Variational mode decomposition based ECG denoising using non-local means and wavelet domain filtering," Australas. Phys. Eng. Sci. Med., vol. 41, no. 4, pp. 891-904, Dec. 2018, doi: 10.1007/s13246-018-0685-0.

[20] Z. Wang, F. Wan, C. M. Wong, and L. Zhang, "Adaptive Fourier decomposition based ECG denoising," Comput. Biol. Med., vol. 77, pp. 195-205, Oct. 2016, doi: 10.1016/j.compbiomed.2016.08.013.

[21] R. R. Sharma and R. B. Pachori, "Baseline wander and power line interference removal from ECG signals using eigenvalue decomposition," Biomed. Signal Process. Control, vol. 45, pp. 33-49, Aug. 2018, doi: 10.1016/j.bspc.2018.05.002.

[22] H. Hao et al., "Multi-lead model-based ECG signal denoising by guided filter," Eng. Appl. Artif. Intell., vol. 79, pp. 34-44, Mar. 2019, doi: 10.1016/j.engappai.2018.12.004.

[23] N. Reljin, J. Lazaro, M. B. Hossain, Y. S. Noh, C. H. Cho, and K. H. Chon, "Using the Redundant Convolutional Encoder-Decoder to Denoise QRS Complexes in ECG Signals Recorded with an Armband Wearable Device,” Sensors, vol. 20, no. 16, Art. no. 16, Jan. 2020, doi: $10.3390 / \mathrm{s} 20164611$

[24] H. Wang, K. Siu, K. Ju, and K. H. Chon, "A High Resolution Approach to Estimating Time-Frequency Spectra and Their Amplitudes," Ann. Biomed. Eng., vol. 34, no. 2, pp. 326-338, Feb. 2006, doi: 10.1007/s10439-005-9035-y.

[25] M.B. Hossain, J. Lázaro, Y. Noh, and K. H. Chon, "Denoising Wearable Armband ECG Data Using the Variable Frequency Complex Demodulation Technique," in 2020 42nd Annual International Conference of the IEEE Engineering in Medicine Biology Society (EMBC), Jul. 2020, pp. 592-595, doi: 10.1109/EMBC44109.2020.9175665.

[26] J. Lázaro, N. Reljin, M. B. Hossain, Y. Noh, P. Laguna, and K. Chon, "Wearable Armband Device for Daily Life Electrocardiogram Monitoring," IEEE Trans. Biomed. Eng., pp. 1-1, 2020, doi: 10.1109/TBME.2020.2987759.

[27] A. L. Goldberger et al., "PhysioBank, PhysioToolkit, and PhysioNet: components of a new research resource for complex physiologic signals," Circulation, vol. 101, no. 23, pp. E215-220, Jun. 2000, doi: 10.1161/01.cir.101.23.e215.

[28] G. B. Moody and R. G. Mark, "The impact of the MIT-BIH Arrhythmia Database," IEEE Eng. Med. Biol. Mag., vol. 20, no. 3, pp. 45-50, May 2001, doi: 10.1109/51.932724.

[29] J. Lázaro, N. Reljin, Y. Noh, P. Laguna, and K. H. Chon, "Feasibility of Long-Term Daily Life Electrocardiogram Monitoring Based on a Wearable Armband Device," in 201941 st Annual International Conference of the IEEE Engineering in Medicine and Biology Society (EMBC), Jul. 2019, pp. 4314-4317, doi: 10.1109/EMBC.2019.8857219.

[30] B. A. Reyes et al., "Novel Electrodes for Underwater ECG Monitoring," IEEE Trans. Biomed. Eng., vol. 61, no. 6, pp. 1863-1876, Jun. 2014, doi: 10.1109/TBME.2014.2309293.

[31] S. K. Bashar, Y. Noh, A. J. Walkey, D. D. McManus, and K. H. Chon, "VERB: VFCDM-Based Electrocardiogram Reconstruction and Beat Detection Algorithm," IEEE Access, vol. 7, pp. 13856-13866, 2019, doi: 10.1109/ACCESS.2019.2894092.

[32] K. H. Chon*, S. Dash, and K. Ju, "Estimation of Respiratory Rate From Photoplethysmogram Data Using Time-Frequency Spectral Estimation,” IEEE Trans. Biomed. Eng., vol. 56, no. 8, pp. 2054-2063, Aug. 2009, doi: 10.1109/TBME.2009.2019766.

[33] H. F. Posada-Quintero, J. P. Florian, Á. D. Orjuela-Cañón, and K. H. Chon, "Highly sensitive index of sympathetic activity based on timefrequency spectral analysis of electrodermal activity," Am. J. Physiol.-Regul. Integr. Comp. Physiol., vol. 311, no. 3, pp. R582-R591, Jul. 2016, doi: 10.1152/ajpregu.00180.2016.

[34] M. Yochum, C. Renaud, and S. Jacquir, "Automatic detection of P, QRS and T patterns in 12 leads ECG signal based on CWT," Biomed. Signal Process. Control, vol. 25, pp. 46-52, Mar. 2016, doi: 10.1016/j.bspc.2015.10.011.

[35] M. B. Hossain, S. K. Bashar, A. J. Walkey, D. D. McManus, and K. H. Chon, “An Accurate QRS Complex and P Wave Detection in ECG Signals Using Complete Ensemble Empirical Mode Decomposition with Adaptive Noise Approach," IEEE Access, vol. 7, pp. 128869128880, 2019, doi: 10.1109/ACCESS.2019.2939943.

[36] H. Zhivomirov, “A Method for Colored Noise Generation,” Romanian J. Acoust. Vib., vol. 15, no. 1, pp. 14-19, Aug. 2018.

[37] M. S. Manikandan and S. Dandapat, "Wavelet energy based diagnostic distortion measure for ECG," Biomed. Signal Process. Control, vol. 2, no. 2, pp. 80-96, Apr. 2007, doi: 10.1016/j.bspc.2007.05.001.

[38] S. K. Mukhopadhyay and S. Krishnan, "A singular spectrum analysis-based model-free electrocardiogram denoising technique," Comput. Methods Programs Biomed., vol. 188, p. 105304, May 2020, doi: 10.1016/j.cmpb.2019.105304.

[39] J. Wang, Y. Ye, X. Pan, and X. Gao, "Parallel-type fractional zero-phase filtering for ECG signal denoising," Biomed. Signal Process. Control, vol. 18, pp. 36-41, Apr. 2015, doi: 10.1016/j.bspc.2014.10.012.

[40] J. Pan and W. J. Tompkins, “A Real-Time QRS Detection Algorithm," IEEE Trans. Biomed. Eng., vol. BME-32, no. 3, pp. 230-236, Mar. 1985, doi: 10.1109/TBME.1985.325532.

[41] J. Lee, B. A. Reyes, D. D. McManus, O. Maitas, and K. H. Chon, "Atrial Fibrillation Detection Using an iPhone 4S," IEEE Trans. Biomed. Eng., vol. 60, no. 1, pp. 203-206, Jan. 2013, doi: 10.1109/TBME.2012.2208112. 